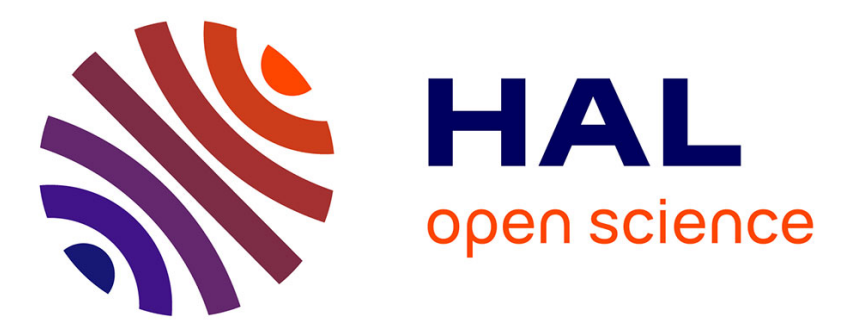

\title{
Confined flow vortex breakdown control using a small rotating disk
}

Louis Mununga, Kerry Hourigan, Mark C. Thompson, Thomas Leweke

\section{To cite this version:}

Louis Mununga, Kerry Hourigan, Mark C. Thompson, Thomas Leweke. Confined flow vortex breakdown control using a small rotating disk. Physics of Fluids, 2004, 16, pp.4750-4753. 10.1063/1.1813061 . hal-00014699

\section{HAL Id: hal-00014699 \\ https://hal.science/hal-00014699}

Submitted on 30 Jun 2020

HAL is a multi-disciplinary open access archive for the deposit and dissemination of scientific research documents, whether they are published or not. The documents may come from teaching and research institutions in France or abroad, or from public or private research centers.
L'archive ouverte pluridisciplinaire HAL, est destinée au dépôt et à la diffusion de documents scientifiques de niveau recherche, publiés ou non, émanant des établissements d'enseignement et de recherche français ou étrangers, des laboratoires publics ou privés. 


\section{Confined flow vortex breakdown control using a small rotating disk}

Cite as: Physics of Fluids 16, 4750 (2004); https://doi.org/10.1063/1.1813061

Submitted: 24 August 2004 . Accepted: 16 September 2004 . Published Online: 11 November 2004

L. Mununga, K. Hourigan, M. C. Thompson, and T. Leweke

\section{ARTICLES YOU MAY BE INTERESTED IN}

Control of vortex breakdown by addition of near-axis swirl

Physics of Fluids 15, 271 (2003); https://doi.org/10.1063/1.1530161

Experimental control of vortex breakdown by density effects

Physics of Fluids 23, 034104 (2011); https://doi.org/10.1063/1.3560386

A study of the geometry and parameter dependence of vortex breakdown

Physics of Fluids 27, 044102 (2015); https://doi.org/10.1063/1.4916352 


\title{
Confined flow vortex breakdown control using a small rotating disk
}

\author{
L. Mununga, ${ }^{a}$ K. Hourigan, and M. C. Thompson \\ Fluids Laboratory for Aeronautical and Industrial Research (FLAIR), Department of Mechanical \\ Engineering, Monash University, P.O. Box 31, Clayton Campus, Victoria 3800, Australia \\ T. Leweke \\ Institut de Recherche sur les Phénomènes Hors Equilibre, UMR 6594 CNRS/Universités Aix-49 \\ rue F. Joliot-Curie-Boite Postale, Marseille, 146, F-13384 Marseille Cedex 13, France
}

(Received 24 August 2004; accepted 16 September 2004; published online 11 November 2004)

\begin{abstract}
As part of a program to investigate the effectiveness of vortex breakdown in bioreactors for cell and tissue growth, a nonintrusive method of flow control is presented. A small rotating disk flush-mounted opposite to the rotating lid in a confined cylindrical vessel is found to precipitate or delay the onset of vortex breakdown depending on whether it is corotating or counterrotating, respectively. Furthermore, corotation increases the bubble radial and axial dimensions while shifting the bubble in the upstream direction. By contrast, counterrotation tends to reduce the size of the bubble, or completely suppress it, while shifting the bubble in the downstream direction. It has also been shown that corotating swirl addition using the small disk is orders of magnitude more energy efficient in manipulating the vortex breakdown bubble than using end wall rotation. (C) 2004 American Institute of Physics. [DOI: 10.1063/1.1813061]
\end{abstract}

Over the years many researchers have attempted to define vortex breakdown, with one of the simplest definitions suggested by Leibovich. ${ }^{1} \mathrm{He}$ described vortex breakdown as a substantial change in the structure of a vortex core as a direct result of a variation in the characteristic ratio of tangential to axial velocity components.

The first observation of vortex breakdown in a cylindrical enclosure with one rotating end wall is credited to Vogel. ${ }^{2}$ His experimental visualizations showed a recirculation region that appeared to resemble a bubble near the axis of rotation. Amongst other important early works on vortex breakdown in confined flows is the detailed investigation by Escudier $^{3}$ who extended the works of Vogel $^{2}$ and Ronnenberg ${ }^{4}$ by mapping regions of one, two, and three vortex breakdowns and regions which are steady and unsteady, as a function of Reynolds number and cylinder aspect ratio. In the past two decades several other studies ${ }^{5,6}$ focused on improving the understanding of the vortex breakdown process have been published.

Vortex breakdown is important in a variety of technical areas such as aerodynamics (open flow application) and combustion. Interestingly, in real-life applications, the presence of vortex breakdown may be detrimental or beneficial. It is therefore of considerable importance to investigate methods of controlling vortex breakdown so that it can be enhanced where advantageous or suppressed where detrimental. Cell or tissue growth research in bioreactors is a very good example of a real-life application of vortex breakdown in confined flow. Current research at Monash University is trying to locate scaffolds in a region of a bioreactor where the flow is laminar, hence with low shear stress, and with a significant

\footnotetext{
${ }^{\text {a) }}$ Author to whom correspondence should be addressed. Telephone: 6103 9905 3544. Fax: 61039905 3807. Electronic mail: lewis.mununga@eng.monash.edu.au
}

residence time for nutrient injection. Such a region can be easily controlled and hence will constitute an ideal environment for cell growth. This laminar and low shear stress region can be provided in form of recirculation zone or vortex breakdown bubble. The method of controlling vortex breakdown being proposed here is an attractive way of providing such a controlled region without excessive additional energy input.

Previously, at least three techniques have been employed to control vortex breakdown in confined flow studies: temperature gradient, ${ }^{7}$ corotation, ${ }^{8,9}$ and counterrotation of the end walls, ${ }^{10}$ and the addition of near-axis swirl. ${ }^{11}$ Recently, Husain et al. ${ }^{11}$ employed the concept of near-axis swirl injection using a small central rod rotating independently of the bottom or rotating end wall. Their findings were that corotation retained steady flow while suppressing vortex breakdown bubbles, whereas counterrotation induced a centrifugal instability resulting in vortex breakdown enhancement. This method, although effective, is intrusive as the rod passes through the center of the vortex core and breakdown bubble.

The overall aim of this study is to evaluate the effectiveness of a proposed new nonintrusive method of controlling vortex breakdown. The research described in this paper investigates whether large changes to the flow structure can be induced by relatively small perturbations to upstream flow. From Leibovich's ${ }^{1}$ definition of vortex breakdown we can deduce that the structure of vortex breakdown can be altered by varying the swirl velocity and axial velocity along the swirl axis. The method suggested here controls vortex breakdown by the addition of swirl (corotating or counterrotating) using a small independently controlled rotating disk (Fig. 1). In addition, the rotation of the small disk also produces an opposing axial flow along the swirl axis. The effects of corotation and counterrotation of the small disk on vortex break- 


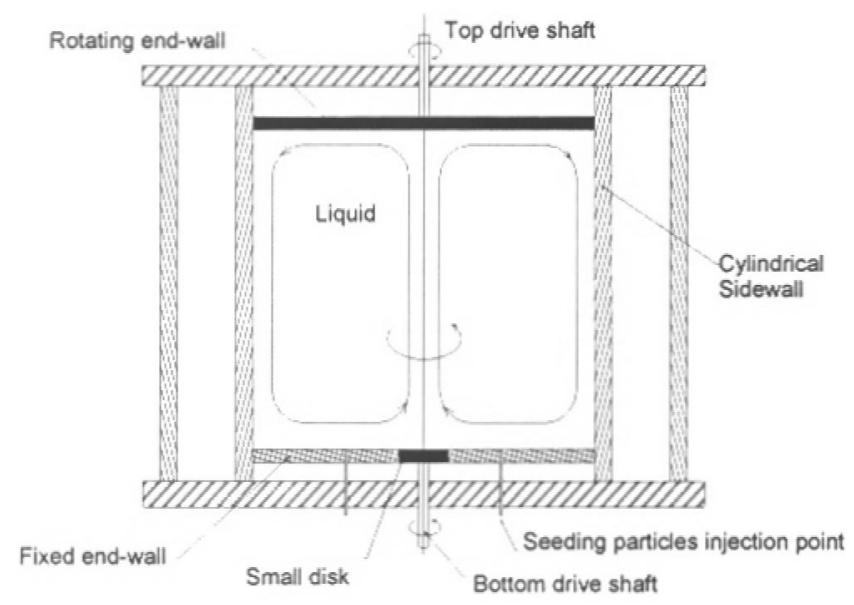

FIG. 1. Experimental rig.

down are examined and quantified leading to a better understanding of the capabilities of this technique.

The experimental rig (Fig. 1) consisted of a cylindrical Perspex tube of radius $R=243 \mathrm{~mm}$ filled with silicone oil of kinematic viscosity $\nu=500 \times 10^{-6} \mathrm{~m}^{2} / \mathrm{s}$ at $25^{\circ} \mathrm{C}$. The aspect ratio $H / R$ was set to 2, where $H$ is height of the fluid column between the two end walls and $R$ is the radius of the cylinder, which is also equal to the radius of the rotating end wall. A small disk of radius $r=0.15 R$, equivalent to $2.25 \%$ of the area of the rotating end wall, was flush mounted concentrically into the nonrotating end wall. The following are the three main dimensionless parameters used to characterize the flow: the angular velocity ratio $\varepsilon=\Omega_{r} / \Omega$, where $\Omega_{r}$ and $\Omega$ are the angular velocities of the small disk and the rotating end wall, respectively; the Reynolds numbers corresponding to the rotating end wall $\operatorname{Re}=\left(\Omega R^{2} / \nu\right)$ and the small disk $\operatorname{Re}_{r}=\left(\Omega r^{2} / \nu\right)$. The flow structure within the working section of the tank was visualized by shining a thin sheet of light $(\approx 5 \mathrm{~mm})$ emanating from a twin 500 watt floodlight. To highlight the flow structures, a small quantity of silicone oil mixed with silver-coated hollow glass spheres $(\approx 10 \mu \mathrm{m}$ diameter) was injected by means of a hypodermic syringe through two small holes in the fixed end wall. Photographs were taken using a 5 megapixel Minolta digital camera with seven times optical zoom.

In the previous-mentioned studies involving the rotation of both end walls of the same size, ${ }^{9,10}$ the angular velocity ratio was restricted to only moderate corotation $(0<\varepsilon<0.5)$ and counterrotation $(-0.5<\varepsilon<0)$, whereas in this study, with a very small control disk, a wider range of rotation ratios $(\varepsilon<-1.0$ and $\varepsilon>1.0)$ are investigated. Initial validation experiments involved only the rotating end wall (i.e., $\varepsilon=0)$ and the visualization results were compared with Escudier's ${ }^{3}$ results for an aspect ratio $H / R=2.0$. In addition to their role in validation, these preliminary results were used to provide reference data for the subsequent study of the bubble size, in the radial and axial directions, as well as its location along the swirl axis. The size and location of the bubble were determined using the image analysis routines of the MATLAB package. The next stage of the study was concerned with investigating the effect of rotation of the control

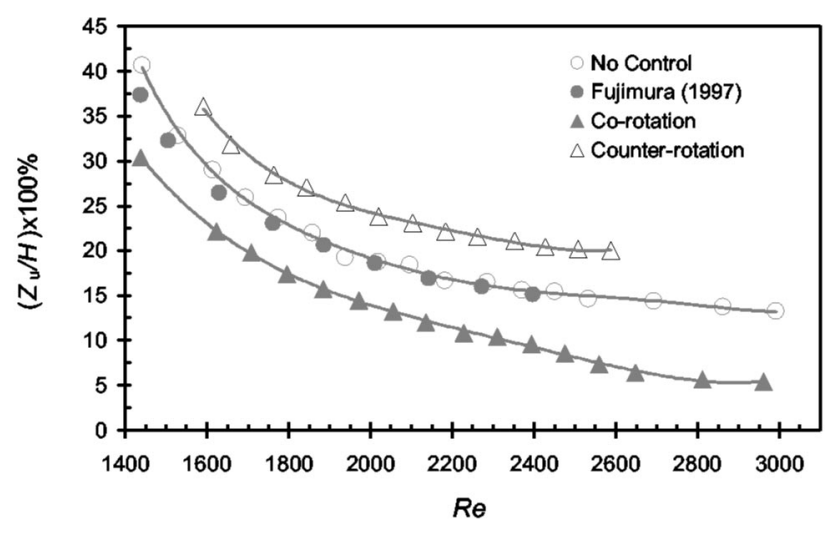

FIG. 2. Variation of the normalized axial location of the upstream stagnation point with Re: No control, corotation and counterrotation at $\operatorname{Re}_{r}=31$.

disk on the bubble size and location using both corotation and counterrotation. In addition, the critical Reynolds number $\left(\operatorname{Re}_{c}\right)$, associated with the first appearance of the bubble, was quantified for different rates of corotation and counterrotation. Selected results from the above experiments are discussed in the following paragraphs.

Figure 2 shows the migration of the first/main vortex breakdown bubble along the axis of rotation by presenting the relative position of the upstream stagnation point scaled by the tank height $\left(Z_{u} / H\right)$. The cases for which the results are shown correspond to no control, here the small disk was stationary; corotation and counterrotation of the small disk at $\operatorname{Re}_{r}=31$, which corresponds to $|\varepsilon|=0.92$ (with respect to $\operatorname{Re}$ =1441). The vortex breakdown bubble was observed to migrate upstream, away from the rotating end wall, as the Reynolds number was increased. The current results, for the "no control" case, agree to within $\pm 2 \%$ with the findings of Fujimura et al., ${ }^{6}$ also reproduced in Fig. 2, for the same aspect ratio $(H / R=2)$. Another important observation in both studies is that, as the Re increases, the rate of change of $Z_{u} / H$ decreases, apparently asymptoting to an almost constant level of $Z_{u} / H=0.13$. The effect of corotation on the location of the upstream stagnation point is to move it further upstream, whereas counterrotation shifts it in the opposite direction. Other results, not shown here, indicate that higher rotation rates (i.e., $\operatorname{Re}_{r}>31$ ) of the control disk result in larger shifts of the upstream stagnation point away from the no control position.

Figure 3 shows experimental results investigating the onset of vortex breakdown, which involved the determination of the critical Reynolds number $\operatorname{Re}_{c}$. Although in reality $\mathrm{Re}_{c}$ is the Reynolds number corresponding to the transition to reversed flow on the axis, for practical reasons in the current investigation it is taken as the lowest Reynolds number tested showing a breakdown bubble. The onset was preceded by the formation of a spiral at a location where the breakdown bubble would eventually appear. A slight increase in $\mathrm{Re}$, beyond this point, resulted in the emergence of a stagnation point upstream of a small recirculation zone (i.e., a vortex breakdown bubble) along the axis of rotation. It is worth noting that the onset of vortex breakdown is associated with a critical balance between the swirl and axial velocities. 


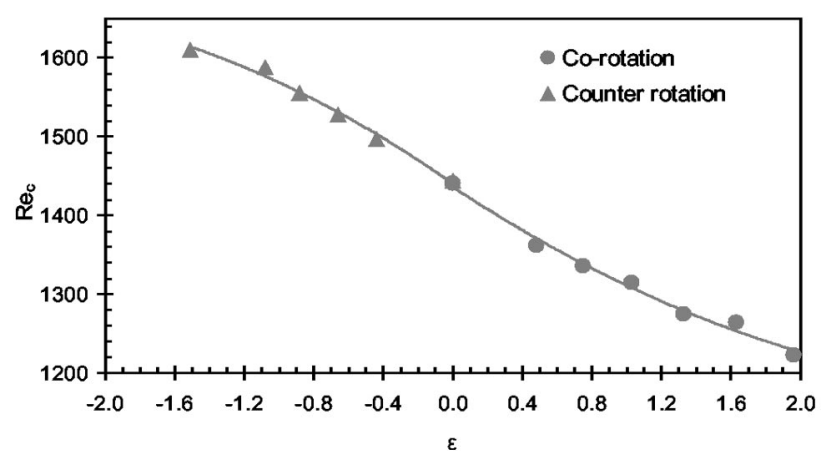

FIG. 3. Variation of the critical Reynolds number $\mathrm{Re}_{c}$ with the rotation ratio, $\varepsilon(>0$, corotation and $<0$, counterrotation).

At the downstream end of the breakdown bubble another stagnation point was observed. In Fig. 3, the relationship between the $\operatorname{Re}_{c}$ and the angular velocity ratio $\varepsilon$ is depicted. In addition, the effects of both corotation and counterrotation on the vortex breakdown onset are shown. It is evident from Fig. 3 that corotation assists the onset of breakdown, as $\operatorname{Re}_{c}$ decreases with increases in $\varepsilon$, whereas counterrotation has the opposite effect. This outcome is consistent with the finding of Bhattacharyya and Pal, ${ }^{9}$ who tested only the case of corotation of two end walls and found that corotation promoted the onset of breakdown. While their investigation involved moderate corotation $(0<\varepsilon<0.5)$ of two end walls, the current research has employed a much smaller disk and extended the rotation ratio range to $-1.6<\varepsilon<2.0$.

The effect of corotation on the vortex breakdown bubble is clearly depicted in Fig. 4. Figure 4(a) shows the onset of the breakdown bubble generated by the rotation of only the rotating end wall $(\operatorname{Re}=1441)$, while Fig. 4(b) reveals the enhancing effect on the size of the breakdown bubble produced by corotating the small disk. It can be observed that an enlarged and upstream shifted bubble is generated as a result of a relatively moderate corotation corresponding to $\varepsilon$ $=0.72$. Figures 4(a) and 4(b) show that this relatively weak corotating swirl injection from the small disk has caused approximately a sixfold increase in the bubble diameter and

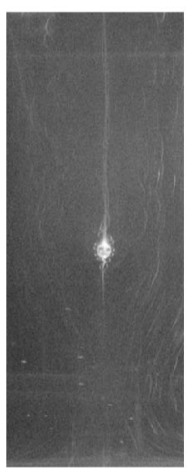

(a)

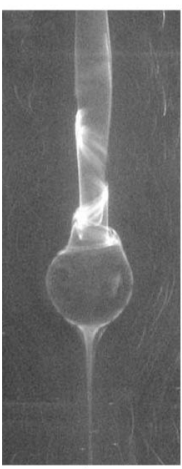

(b)

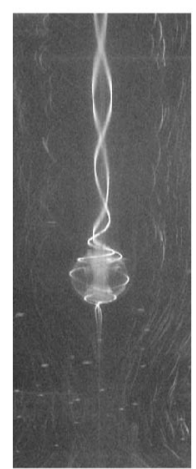

(c)

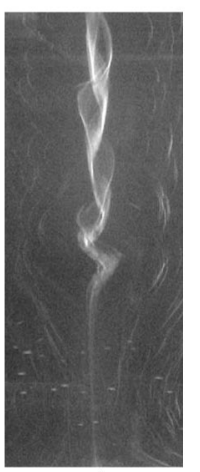

(d)
FIG. 4. Effect additional swirl by the small disk rotation on the breakdown bubble: (i) $\operatorname{Re}=1441$ (a) $\varepsilon=0$ and (b) corotation at $\varepsilon=0.72$, (ii) $\operatorname{Re}=1507$ (c) $\varepsilon=0$ and (d) counterrotation at $\varepsilon=-0.92$. Note: only the central region of the test section is shown. a fivefold increase in the axial dimension. This result supports the thesis that vortex breakdown can be enhanced by injecting a small amount of corotating swirl using a small disk.

Figure 4(b) shows that the effect of corotation is to stretch the vortex breakdown bubble in both the axial and radial directions while making the asymmetry at the downstream end of the bubble become more apparent. It is worth noting that even the bubble generated by the rotating end wall alone [Fig. 4(c)] exhibits some degree of asymmetry at the downstream end, which can be explained by the findings of Thompson and Hourigan. ${ }^{12}$ They reported that even minute imperfections in the experimental apparatus could lead to significant asymmetries in the breakdown bubble; hence breakdown bubbles observed in axisymmetric experiments are almost always going to have some degree of asymmetry.

Other results, not reported in this paper, reveal that the center of the vortex breakdown bubble maintained a nearly constant level regardless of the intensity of additional swirl, although it appears to drop by a fraction in the initial stage (i.e., at very low values of corotation ratio). This shows that corotation does not significantly affect the overall location of the bubble, although it enhances its size. The downstream stagnation point migrates in the downstream direction, as opposed to the upstream stagnation point movement (Fig. 2). Both stagnation points shift further away as $|\varepsilon|$ increases.

Of course, it is not surprising that modifying the upstream swirl distribution will alter the breakdown bubble properties. An important question is whether the perturbation is in some sense small relative to the effect. A partial answer is provided by examining the increase in the power input to the fluid to increase the bubble size from that shown in Fig. 4(a), at the onset of vortex breakdown, to that shown in Fig. 4(b). To produce the bubble shown in Fig. 4(b) involved an increase in the rotational speed of the rotating end wall of about $6 \%$, which in turn corresponds to an increase in the input power by $15 \%$. On the other hand, using the corotation of the small disk at $\varepsilon=0.72$ resulted in the generation of vortex breakdown bubble of roughly the same size as that depicted in Fig. 4(b), while causing an additional power input of only about $0.026 \%$. These input power values are based on some preliminary results from a parallel computational program, the details of which will be presented in follow-up papers. The above analysis shows that it is about 500 times more economical to produce the vortex breakdown bubble shown in Fig. 4(b) using a small disk corotation than using only the end wall rotation. This calculation shows one of the attractive features of the control technique-its energy efficiency. Apart from this, it demonstrates that it is much more effective to control vortex breakdown in confined cylinders by manipulating conditions immediately upstream of the breakdown site. Of course, this has been realized for many years for controlling vortex breakdown in open flow systems (e.g., Refs. 13 and 14), although the control is usually exercised through blowing or suction.

In some situations it is desirable to suppress the occurrence of breakdown, rather than enhance the size or manipulate the features of the breakdown bubble. Counterrotation of 
the small disk can reduce the size of, and in some cases completely suppress, the vortex breakdown bubble. A comparison between Figs. 4(c) and 4(d) clearly illustrates the effect of additional counterrotating swirl on the vortex breakdown bubble. The vortex breakdown bubble in Fig. 4(c) was generated by rotating the end wall at $\mathrm{Re}=1507$ with no control, while Fig. 4(d) depicts the disappearance of the bubble following counterrotation of the small disk at $\varepsilon=-0.92$. Other results (not reproduced here) indicate that the larger the bubble the higher the rotation ratio required to completely destroy it. Based on these results, it can be concluded that a small disk, the area of which is $2.25 \%$ relative to the rotating end wall, is able to generate a counterrotating swirl and opposing axial flow along the axis of rotation strong enough to completely suppress vortex breakdown. As was the case with the corotating disk, this can be done with relatively little power input. The upstream stagnation point and the center of the breakdown bubble migrate downstream with increasing rotation rate, whereas the downstream stagnation point maintains a near constant position except at lower values of the rotation ratio. This is different from the behavior observed for the corotating case, where the center of the bubble maintained an almost constant position.

In conclusion, a nonintrusive method of controlling vortex breakdown has been presented. It has been shown that corotation and counterrotation of the small disk are both effective and efficient in controlling vortex breakdown. The controlling effect is due to the injection of corotating and counterrotating swirl and also the generation of an opposing axial flow induced by the centrifugal action of the rotating disk. These act to modify the flow characteristics of the main vortex core upstream of the vortex breakdown-forming region. Results for the onset of breakdown, using this approach, have revealed that corotation of the small disk precipitates vortex breakdown formation while counterrotation delays or even completely suppresses breakdown. An important result is that corotating swirl addition using the small disk is more energy efficient in enhancing vortex breakdown than end wall rotation. The fact that this work has success- fully demonstrated the ability to control vortex breakdown without (intrusively) interfering with the core vortex can lead to finding practical solutions of some industrial applications where this phenomenon is an important factor. In particular, current research on mixing in bioreactors, which is exploring ways to create and manipulate a controlled environment with low shear stress for cell growth, can benefit from the results of this work. The much needed controlled environment can be achieved by enhancing vortex breakdown formation using the present technique.

${ }^{1}$ S. Leibovich, "The structure of vortex breakdown," Annu. Rev. Fluid Mech. 10, 221 (1978).

${ }^{2}$ H. U. Vogel, "Experimentelle Ergebnisse über die laminare Strömung in einen zylindrischen Gehäuse mit darin rotierender Scheibe," Max-PlanckInstitut, Bericht 6 (1968).

${ }^{3} \mathrm{R}$. Escudier, "Observations of the flow produced in a cylindrical container by a rotating endwall," Exp. Fluids 2, 189 (1984).

${ }^{4}$ B. Ronnenberg, "Ein selbstjustierendes 3-KomponentenLaserdoppleranemometer nach dem Vergleichsverfahren, angewandt auf Untersuchungen in einer stationaren zylinder-symmetrischen Drestromung mit einem Ruckstromgebeit," Max-Planck-Institut für Strömungsforschung, Bericht 20 (1977).

${ }^{5}$ S. Leibovich, "Vortex stability and breakdown: Survey and extension," AIAA J. 22, 1193 (1984).

${ }^{6}$ K. Fujimura, H. S. Koyama, and J. M. Hyun, "Time-dependent vortex breakdown in a cylinder with a rotating lid," ASME J. Fluids Eng. 119, 450 (1997).

${ }^{7}$ M. A. Herrada and V. Shtern, "Control of vortex breakdown by temperature gradients," Phys. Fluids 15, 3468 (2003).

${ }^{8}$ D. T. Valentine and C. C. Jahnke, "Flows induced in a cylinder with both end walls rotating," Phys. Fluids 6, 2702 (1994).

${ }^{9}$ S. Bhattacharyya and A. Pal, "Axisymmetric vortex breakdown in a filled cylinder," Int. J. Eng. Sci. 36, 555 (1998).

${ }^{10}$ K. G. Roesner, "Recirculating zones in a cylinder with rotating lid," Topo. Fluid Mech. Proceeding of IUTAM Symposium, Cambridge, 1990.

${ }^{11}$ H. S. Husain, V. Shtern, and F. Hussain, "Control of vortex breakdown by addition of near-axis swirl," Phys. Fluids 15, 271 (2003).

${ }^{12}$ M. C. Thompson and K. Hourigan, "The sensitivity of steady vortex breakdown bubbles in confined cylinder flows to rotating lid misalignment," J. Fluid Mech. 496, 129 (2003).

${ }^{13}$ A. M. Mitchell and J. Delery, "Research into vortex breakdown control," Prog. Aerosp. Sci. 37, 385 (2001).

${ }^{14}$ W. Gu, O. Robinson, and D. Rockwell, "Control of vortices on a delta wing by leading-edge injection,” AIAA J. 31, 1177 (1993). 